\title{
Noninvasive detection of functional brain activity with near-infrared diffusing-wave spectroscopy
}

Jun Li

Universität Konstanz

Fachbereich Physik and Fachbereich Psychologie

Universitätsstr. 10

78457 Konstanz, Germany

\section{Gregor Dietsche \\ Universität Konstanz \\ Fachbereich Physik \\ 78457 Konstanz, Germany}

\author{
Diana Iftime \\ Universität Konstanz \\ Fachbereich Psychologie \\ 78457 Konstanz, Germany
}

\author{
Sergey E. Skipetrov \\ Université Joseph Fourier \\ Laboratoire de Physique et Modélisation des Milieux \\ Condensés, CNRS \\ 38042 Grenoble, France
}

\section{Georg Maret}

Universität Konstanz

Fachbereich Physik

78457 Konstanz, Germany

\begin{abstract}
We use near-infrared dynamic multiple scattering of light [diffusing-wave spectroscopy (DWS)] to detect the activation of the somato-motor cortex in 11 right-handed volunteers performing a finger opposition task separately with their right and left hands. Temporal autocorrelation functions $g^{(1)}(\mathbf{r}, \tau)$ of the scattered light field are measured during 100-s periods of motor task alternating with 100-s resting baseline periods. From an analysis of the experimental data with an analytical theory for $g^{(1)}(\mathbf{r}, \tau)$ from a three-layer geometry with optical and dynamical heterogeneity representing scalp, skull, and cortex, we obtain quantitative estimates of the diffusion coefficient in cortical regions. Consistent with earlier results, the measured cortical diffusion coefficient is found to be increased during the motor task, with a strong contralateral and a weaker ipsilateral increase consistent with the known brain hemispheric asymmetry for right-handed subjects. Our results support the interpretation of the increase of the cortical diffusion coefficient during finger opposition being due to the functional increase in cortical blood flow rate related to vasodilation.
\end{abstract}

Keywords: noninvasive imaging; optical brain imaging; diffusing-wave spectroscopy.

\section{Thomas Elbert \\ Brigitte Rockstroh \\ Universität Konstanz \\ Fachbereich Psychologie \\ 78457 Konstanz, Germany}

\section{Thomas Gisler}

Universität Konstanz

Fachbereich Physik

78457 Konstanz, Germany

E-mail: thomas.gisler@uni-konstanz.de

\section{Introduction}

Optical methods have become increasingly important as diagnostic tools for noninvasive biomedical imaging, since they offer great advantages to established methods such as positron emission tomography and magnetic resonance imaging in terms of portability, price, and weakness of interaction (absence of radiation damage, etc.). ${ }^{1,2}$ In particular, near-infrared light at wavelengths $\lambda$ between around 750 and $950 \mathrm{~nm}$ provides significant advantages over visible or infrared light, since absorption by the most abundant chromophores in tis-

Address all correspondence to Thomas Gisler, Fachbereich Physik, Universität Konstanz, Universitätsstr. 10, 78457 Konstanz, Germany. Tel: +49-7531-88 3868. Fax: +49-7531-88 3090. E-mail: thomas.gisler@uni-konstanz.de sue, such as melanin and hemoglobin, or water, is weak, while at the same time scattering mean free paths are long enough that light can penetrate up to a few centimeters into tissue. However, the strong scattering of light is a generic obstacle to direct imaging of subsurface features in the body. It has its origin in fluctuations of the dielectric constant on the length scale of the wavelength of light, associated with structural elements such as collagen fibers, cell membranes, and subcellular organelles. Rather than ballistic, light propagates diffusively deep inside tissue, with a photon diffusion coefficient $\mathcal{D}=c l^{*} / 3$ determined by the speed of light in the medium $c$ and the transport mean free path length $l^{* 3}$. This quantity is 
roughly the distance over which scattering completely randomizes the direction of an incoming photon. Diffusive light propagation in tissue thus strongly reduces the spatial resolution of optical imaging methods, in particular for structures deep within the body.

Several methods are currently used to extract, from the distribution of photon fluxes measured at the surface, information on subsurface structures with a scattering or absorption contrast: 1 . time-of-flight experiments with gated detection allow for the discrimination of the early arriving ballistic photons and the reconstruction of optical density distributions $;{ }^{4}$ 2. low-coherence tomography operates in reflection and allows to probe near-surface structures with a longitudinal resolution as small as several $\mu \mathrm{m} ;{ }^{5} 3$. photon migration imaging (PMI) uses a light source modulated at megahertz frequencies and measures the attenuation and retardation of the diffusely transmitted light intensity that is detected some distance away from the source. ${ }^{6}$ Photon migration imaging requires some means of calculating distributions of optical densities and absorption cross sections within the volume from an inversion of the measured data. ${ }^{7}$

PMI has recently been applied to noninvasive functional imaging of cerebral activity after stimulation of specific brain areas such as the visual and motor cortices. Using a backprojection method, low-resolution maps of cortical blood oxygenation following motor stimulation have been obtained with a temporal resolution of $160 \mathrm{~ms}^{8-10}$ These experiments show that the total hemoglobin concentration increases within about $4 \mathrm{~s}$ after the stimulation by up to $0.3 \mu \mathrm{M}$ above its average level of about 70 to $80 \mu \mathrm{M}$. At much shorter times after stimulation (tens of milliseconds), PMI experiments have revealed small stimulus-related signal changes that are interpreted as an increase in the cortical turbidity. ${ }^{11-13}$ This fast optical signal had previously been observed in reflectance measurements on exposed cortical areas. ${ }^{14,15}$ Since the time scale of the fast optical signal is too short for significant optical changes due to increased blood flow, it is believed that it reflects the increased scattering from nerve cells following electrical activation, as has been directly observed in isolated neurons. ${ }^{16}$ Increased activation-related scattering, in turn, is attributed to mechanisms such as cell swelling, increased concentration of vesicles, and ion density gradients. ${ }^{17}$

In addition to the attenuation and retardation of the diffusely transmitted light intensity measured in PMI, a wealth of information is contained in the speckle pattern of multiple scattered light, which forms when a light source with a large coherence length is used. This speckle pattern is a complicated interferogram arising from the coherent superposition of multiple scattered electric fields at the detector. It contains information on the positions of all scatterers, such as cell membranes, erythrocytes, and mitochondria, in the volume swept by the diffuse photon cloud. Similar to the situation in a simple interferometer, motions of the scatterers (due to, e.g., shear flow or Brownian motion) impose changes in the phases of the scattered electric field $E(\mathbf{r}, t)$ at the position $\mathbf{r}$ and time $t$, which leads to temporal fluctuations of the detected intensity. The intensity $I(\mathbf{r}, t)=|E(\mathbf{r}, t)|^{2}$ of the surface speckle pattern thus rapidly fluctuates at locations whose underlying dynamics are fast, while regions with slow dynamics will result in slow fluctuations of the surface speckle pattern. From spa- tially resolved measurements of the surface speckle contrast using, e.g., a CCD camera, time-resolved maps of the superficial blood flow in cortical tissue can be obtained. ${ }^{18}$ This technique has been used for the study of hemodynamic changes in exposed rat brains during cortical spreading depression $^{19,20}$ and somato-sensory activation. ${ }^{21}$

On the other hand, measurements of the temporal autocorrelation function $g^{(1)}(\mathbf{r}, \tau)=\left\langle E^{*}(\mathbf{r}, t) E(\mathbf{r}, t+\tau)\right\rangle /\left\langle|E(\mathbf{r}, t)|^{2}\right\rangle$ of the fluctuating electric field amplitude $E(\mathbf{r}, t)$ of the speckle pattern yields detailed information on the mean-squared displacement $\left\langle\Delta r^{2}(\tau)\right\rangle$ of scatterers within the time $\tau$ by the relation $^{3,22}$

$$
g^{(1)}(\mathbf{r}, \tau)=\int_{l^{*}}^{\infty} P(\mathbf{r}, s) \exp \left[-\frac{1}{3} k_{0}^{2}\left\langle\Delta r^{2}(\tau)\right\rangle \frac{s}{l^{*}}\right] \mathrm{d} s .
$$

Here, $k_{0}=2 \pi / \lambda$ is the wavenumber of light in the medium, and $P(\mathbf{r}, s)$ is the normalized distribution of photon path lengths $s$ at the position $\mathbf{r}$ for a source located at the origin. The latter quantity can be measured with a time-of-flight experiment. Diffusing-wave spectroscopy (DWS), as this multiple-scattering counterpart of quasi-elastic light scattering is called, differs from the latter in that the accumulated phase shift of a photon undergoing $s / l^{*}$ scattering events results in a decay of $g^{(1)}(\mathbf{r}, \tau)$ for single-particle displacements $\Delta r$ $\sim \lambda /(2 \pi)\left(l^{*} / s\right)^{1 / 2}$ that can be much smaller than the wavelength $\lambda$ of light due to the long path length $s \gg l^{*}$. For $l^{*}$ $=1 \mathrm{~mm}$ and a source-receiver distance $L=20 \mathrm{~mm}$, the average number of scattering events is roughly $\left(L / l^{*}\right)^{2}=400$, leading to resolvable displacements $2 \mathrm{~nm}<\Delta r<20 \mathrm{~nm}$ for $\lambda$ $=800 \mathrm{~nm}$. On the other hand, the fact that the long photon paths give rise to the decay of $g^{(1)}(\mathbf{r}, \tau)$ at short times (and, conversely, the short photon paths to the long-time decay) conveys DWS a sort of coarse spatial resolution. In addition, the shape of the autocorrelation function is sensitive to the type of microscopic motion giving rise to speckle fluctuations, allowing, for instance, to discriminate laminar or shear flows from isotropic Brownian motion. ${ }^{23-25}$ The signature of the particle dynamics in the measured autocorrelation function can be used to detect dynamical heterogeneities within a turbid medium, even when scattering or absorption contrasts are entirely absent. ${ }^{26-28}$ Measuring $g^{(1)}(\mathbf{r}, \tau)$ at different positions on the surface of the turbid medium then allows us to coarsely localize dynamical heterogeneities that are buried as deep as $10 l^{*}$ within the opaque bulk medium.

Experiments using DWS for brain imaging are still relatively scarce. Aiming at mapping blood flow velocities in cortical vessels, Lohwasser and Soelkner have tried to account for the effects of multiple light scattering in laser Doppler spectra from brain phantoms. ${ }^{29}$ DWS experiments by Cheung et al. on a semi-exposed rat brain revealed that the field autocorrelation function shows a faster decay on hypercapnia. ${ }^{30}$ The interpretation in terms of increased flow velocity of erythrocytes in the cortical vessels was also supported by parallel NIRS and DWS experiments on a rat brain during focal ischemia. ${ }^{31}$ Using motor cortex stimulation by finger opposition, Durduran et al. ${ }^{32}$ were able to show that a similar acceleration of $g^{(1)}(\mathbf{r}, \tau)$ can be measured fully noninvasively over the human motor cortex. An analysis of the DWS data with a semi-infinite one-layer model, using an empirical correction 
factor accounting for the underestimation of the cortical diffusion coefficient by the one-layer model, resulted in functional increases of the cortical dynamics of about $40 \%$, which were interpreted due to the functional enhancement of the cortical blood flow rate.

While the shape of the measured field autocorrelation functions reported by Cheung et al. ${ }^{30}$ deviates from predictions for simple diffusion or random flow, the correction factor used by Durduran et al. ${ }^{32}$ suggests that using DWS to quantitatively determine cortical perfusion rates in an entirely noninvasive way is likely to be complicated by the heterogeneous optical and dynamical properties of the head consisting of scalp, skull, cerebrospinal fluid, and gray and white matter. Model calculations of autocorrelation functions from optically and dynamically heterogeneous multilayer media based on the solution of the correlation-diffusion equation do indeed show a complicated decay of $g^{(1)}(\mathbf{r}, \tau)$, which does not factorize into contributions from the individual layers. ${ }^{33}$

We show that the DWS autocorrelation functions measured from the human motor cortex through intact scalp and skull can be analyzed quantitatively with a three-layer model allowing for different optical and dynamical parameters in the scalp, skull, and cortex. This allows for separating the cortical dynamics from tissue optical parameters and scalp dynamics reflecting peripheral perfusion. For right-handed subjects, we find a significant increase of the cortical diffusion coefficient when a finger opposition task is performed. In accordance with the enhanced activation of the contralateral hand area of the primary somato-sensory and primary motor cortex by the finger opposition task, ${ }^{34-37}$ we find that the cortical diffusion coefficient for contralateral (right-hand) stimulation is significantly higher than the one for ipsilateral (left-hand) stimulation. An analysis of the possible origins of the observed increase in the cortical diffusion coefficient indicates that for the present motor stimulation protocol, the DWS signal is mainly dominated by functional increases of blood flow rate and less by increases in the blood volume, in accordance with the conclusions arrived at by Durduran et al. ${ }^{32}$

\section{Methods}

\subsection{Subjects}

11 healthy adult volunteers (four male, mean age 30.5 years, range 22 to 53 years) were recruited from students and faculty of the University of Konstanz. All subjects were right handed as verified by a modified version of the Edinburgh Handedness Questionnaire. ${ }^{38}$ The average score was 80 to 100; a 12th subject was discarded as left handed. Prior to the experiment, subjects were informed about the task and the measuring procedure and signed a written consent. The study protocol was approved by the University's Ethical Review Board.

\subsection{Task and Procedure}

During task and measurements, the subject was sitting on a chair in a relaxed position and was asked to perform a finger opposition task with the right (dominant) hand. Task periods lasting $100 \mathrm{~s}$ each followed rest periods of $100 \mathrm{~s}$, during which the baseline data were recorded. Afterward, the sequence of baseline and task periods was repeated with the left hand. Subjects were asked to perform the finger opposition as

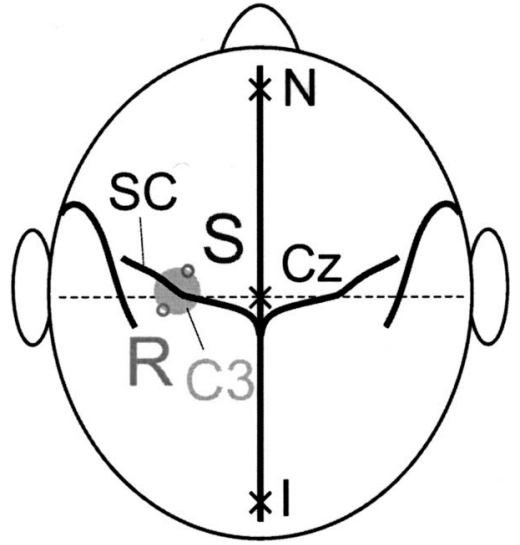

Fig. 1 Experimental geometry for the DWS experiments. $\mathrm{N}$ is nasion, $\mathrm{I}$ is inion, $\mathrm{Cz}$ is vertex, and SC is central sulcus. Source and receiver fibers (denoted by $S$ and $R$, respectively) are placed over the $\mathrm{C} 3$ area of the somato-sensory cortex, approximately perpendicular to the central sulcus. For the right-handed subjects investigated here, finger opposition with the left hand corresponds to ipsilateral stimulation, and right-hand finger opposition to contralateral stimulation.

fast and vigorously as possible, avoiding repetitive alternations. They were further instructed that they might imagine playing a piano or a violin to increase the similarity to motor activation in real life.

\subsection{Diffusing-Wave Spectroscopy}

A diode laser operating at a wavelength $\lambda_{0}=802 \mathrm{~nm}$ (Toptica TA100) was coupled into a multimode optical fiber (core diameter $50 \mu \mathrm{m}$; numerical aperture 0.22) serving as an illuminating source. Multiple scattered light was collected by a fewmode optical fiber guiding about six transverse modes ${ }^{39}$ at a distance of 20 to $25 \mathrm{~mm}$ from the source and detected with an avalanche diode operating in Geiger mode (Perkin-Elmer SPCM-AQR-15-FC). The intensity autocorrelation function $g^{(2)}(\mathbf{r}, \tau)=\langle I(\mathbf{r}, t) I(\mathbf{r}, t+\tau)\rangle /\langle I(\mathbf{r}, t)\rangle^{2}$ was then computed from the amplified and discriminated detector output by a digital multitau correlator (ALV5000E) at lag times between $200 \mathrm{~ns}$ and $3.1 \mathrm{~s}$. Source and receiver fibers were mounted on a rigid fixation device that the subject could wear like a helmet. The intensity of the illuminating light was adjusted to be less than the maximum allowable intensity of $4 \mathrm{~mW} / \mathrm{mm}^{2} .40$ Photon count rates of 20 to $80 \mathrm{kHz}$ high enough for computation of intensity autocorrelation functions within the measurement period of $100 \mathrm{~s}$ were obtained by increasing both the incident laser power and the illuminated area on the scalp, thereby still keeping the peak intensity below the safety limit. This procedure was especially important for measurements on strongly pigmented subjects. The sensor, comprising light source and detector, was then fixed over the motor area on the left hemisphere [area C3 in the international 10-20 system for electroencephalography $\left.(\mathrm{EEG})^{41}\right]$, using vertex and ears as external landmarks (see Fig. 1). The direction from source to detector was arranged perpendicular to the direction of the central sulcus to facilitate the photon cloud to cross the area activated most by the motor task. Before fixing the source and detector, the hair was combed away so that the illuminator and detector had good optical contact to the scalp. During both the resting baseline and the task periods, each lasting 
$100 \mathrm{~s}$, we recorded ten normalized intensity autocorrelation functions, each with a duration of $10 \mathrm{~s}$. To make sure that the amplitude of the measured intensity autocorrelation function was due to the few-mode receiver optics only (and not due to contributions of static scattering to the signal ${ }^{42}$ ), we measured $g^{(2)}(\mathbf{r}, \tau)$, using the same setup, from a turbid latex sample immediately before and after the measurements on the subjects.

\subsection{Data Analysis}

We model the head by a simplified three-layer geometry consisting of scalp (layer 1), skull (layer 2), and cortex (layer 3), each of them characterized by its thickness $\Delta_{n}$, transport mean free path length $l_{n}^{*}$, absorption path length $l_{n}^{(\mathrm{a})}$, and diffusion coefficient $D_{n}(n=1,2,3)$. The cortex was assumed to be infinitely thick.

We used a Levenberg-Marquardt optimization routine to determine the diffusion coefficients $D_{1}$ and $D_{3}$ of the scalp and the brain, respectively, from fitting the analytical expression for the field autocorrelation function $g^{(1)}(\mathbf{r}, \tau)$ for this three-layer model (see Appendices 1 and 2) to the measured intensity autocorrelation functions $g^{(2)}(\mathbf{r}, \tau)$, using the Siegert relation $^{43} g^{(2)}(\mathbf{r}, \tau)=1+\beta_{\text {coh }}\left|g^{(1)}(\mathbf{r}, \tau)\right|^{2}$ between field and intensity autocorrelation functions. The other parameters, such as the absorption lengths $l_{1,2,3}^{\text {(a) }}$, transport mean free path lengths $l_{1,2,3}^{*}$, and the thicknesses $\Delta_{1}$ and $\Delta_{2}$ of the scalp and of the skull, respectively, were estimated from the baseline data by optimization within a bounded range, using the litera-

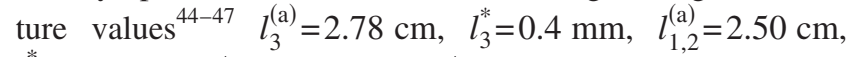
$l_{1,2}^{*}=0.5 \mathrm{~mm}, \Delta_{1}=2 \mathrm{~mm}$, and $\Delta_{2}=5 \mathrm{~mm}$ as initial guesses. For the analysis of the data recorded during stimulation phases, we fixed the values of $l_{1,2,3}^{(\mathrm{a})}, l_{1,2,3}^{*}, \Delta_{1}$, and $\Delta_{2}$ to their baseline values. We approximated the skull as a static medium (i.e., $D_{2}=0$ ). This allowed us to reduce the free parameters in the fitting of the stimulation data to the diffusion coefficients $D_{1}, D_{3}$.

The stability and accuracy of the fitting procedure was tested on simulated field autocorrelation functions obtained from Monte-Carlo simulations of our three-layer model. The diffusion coefficients of scalp and cortex obtained from fitting the analytic three-layer theory to the simulated data were found to agree to within $5 \%$ for $D_{1}$ and $2 \%$ for $D_{3}$ with the true values used for generating the data.

Effects of activation were evaluated by comparing the diffusion coefficients measured during stimulation and during rest periods for both contralateral (right hand) and ipsilateral (left hand) stimulation by Student's $t$-test.

\section{Results}

Figure 2 shows the reduced intensity autocorrelation functions $\left[g^{(2)}(\mathbf{r}, \tau)-1\right] / \beta_{\mathrm{coh}}$ from a selected subject obtained from the left hemisphere (C3) during baseline and activation, respectively. Data are averages over two blocks of ten runs each. The autocorrelation function recorded during contralateral stimulation shows a significantly faster decay than during the resting baseline period. Modeling the autocorrelation function by the solution of the correlation-diffusion equation for a semi-infinite one-layer geometry, assuming free diffusion as the mechanism for speckle decorrelation, gave rather poor agreement with the experimental data. Modeling the dynamics by a random flow $\left\langle\Delta r^{2}(\tau)\right\rangle=\left\langle v^{2}\right\rangle \tau^{2}$ with a Gaussian flow velocity $v$ yielded, due to the increased curvature of $g^{(1)}(\mathbf{r}, \tau)$, even poorer agreement than the free diffusion model. These discrepancies are resolved when we use the solution of the correlation-diffusion Eq. (2) for a three-layer medium, representing scalp and skull by finite layers and the cortex as the innermost, semi-infinite layer. With this dynamic three-layer model, the fitting procedure yields a cortical diffusion coefficient $D_{3}=4.9 \times 10^{-9} \mathrm{~cm}^{2} / \mathrm{s}$ during the resting period and $D_{3}=1.3 \times 10^{-8} \mathrm{~cm}^{2} / \mathrm{s}$ during the stimulation period. The best-fit value $\Delta_{2}=4.7 \mathrm{~mm}$ for the skull thickness obtained from the DWS data was, to within experimental error, identical for both baseline and stimulation data.* Although the skull was modeled as a completely static layer, its incorporation was found to be necessary to account for the decay of the autocorrelation function at intermediate times $20 \mu \mathrm{s} \leqslant \tau$ $\leqslant 50 \mu$ s. The decay of the autocorrelation function at long times is dominated by the scalp diffusion coefficient $D_{1}$, which is found to increase with stimulation (see below).

When measuring the autocorrelation function after the end of the motor task, the correlation functions were found to be identical with the baseline correlation functions before stimulation. Analyzing the individual autocorrelation functions from two blocks yields information on the distribution of the fitting parameters $D_{1}$ and $D_{3}$. Due to the limited photon statistics and decay times in the 0.1-ms range, individual autocorrelation functions recorded over $10 \mathrm{~s}$ are noisier than the averaged autocorrelation function. Nevertheless, the fitting routine converges, and, using a fixed skull thickness $\Delta_{2}$ $=4.7 \mathrm{~mm}$, the average best-fit values $D_{1}=(1.1 \pm 0.3)$ $\times 10^{-9} \mathrm{~cm}^{2} / \mathrm{s}$ and $D_{3}=(4.7 \pm 1.2) \times 10^{-9} \mathrm{~cm}^{2} / \mathrm{s}$ for the baseline, and $D_{1}=(1.9 \pm 0.5) \times 10^{-9} \mathrm{~cm}^{2} / \mathrm{s}$ and $D_{3}=(1.4 \pm 0.5)$ $\times 10^{-8} \mathrm{~cm}^{2} / \mathrm{s}$ for stimulation, agree well with the diffusion coefficients obtained from fitting the averaged data. Furthermore, this analysis shows that the differences in $D_{3}$ and $D_{1}$ on motor stimulation observed in the averaged autocorrelation functions are indeed significant.

When the stimulation is exerted by the left hand, ipsilateral to $\mathrm{C} 3$, the autocorrelation function recorded from $\mathrm{C} 3$ is found to decay significantly more slowly than for contralateral stimulation (see Fig. 3). This slower decay is mainly due to the fact that the cortical diffusion coefficient for contralateral stimulation $D_{3}=1.3 \times 10^{-8} \mathrm{~cm}^{2} / \mathrm{s}$ is larger than the one for ipsilateral stimulation $D_{3}=9.8 \times 10^{-9} \mathrm{~cm}^{2} / \mathrm{s}$. In contrast, the scalp diffusion coefficients are not significantly different between ipsilateral and contralateral stimulation, although they are both increased with respect to the value for the resting period.

Autocorrelation functions obtained from the 11 subjects measured during resting and stimulation periods showed equally good agreement with the predictions of the correlation-diffusion theory. The absolute values of the cortical diffusion coefficient were found to vary over more than a decade within the group of 11 subjects, both for the baseline and stimulation periods. However, normalizing the value of the cortical diffusion coefficient measured during stimulation

*This value is in good agreement with the skull thickness of $(4.7 \pm 1.0) \mathrm{mm}$ determined by magnetic resonance imaging. 


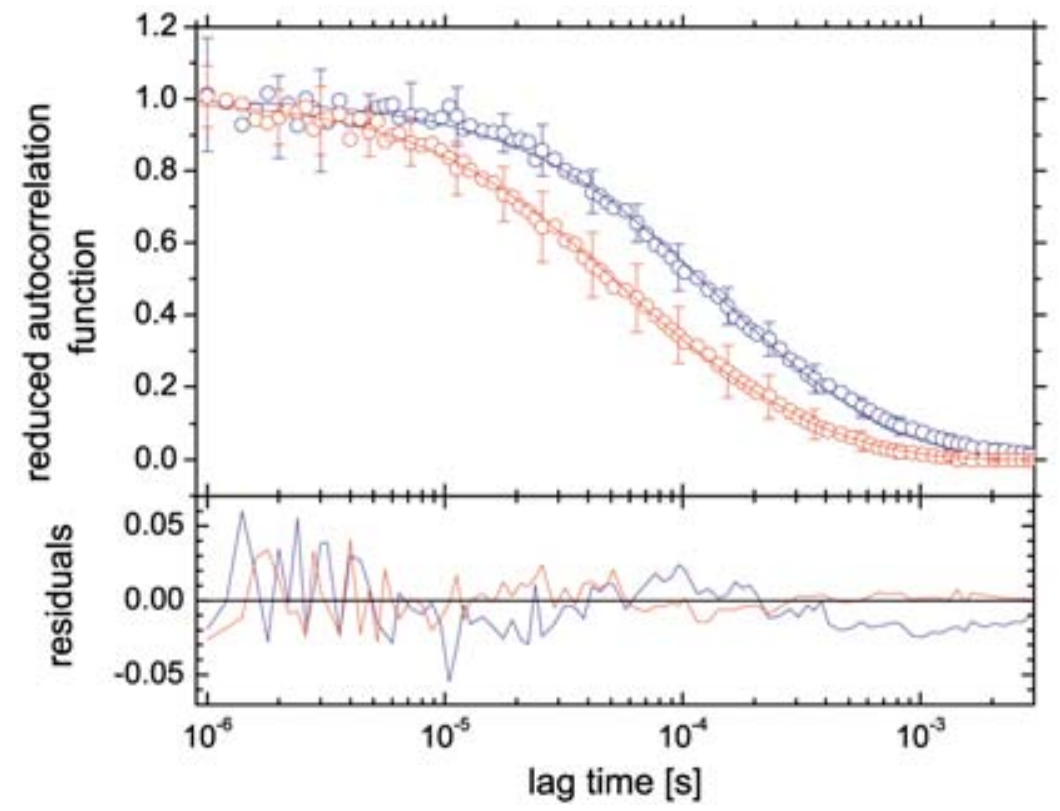

Fig. 2 Reduced intensity autocorrelation function $\left[g^{(2)}(r, \tau)-1\right] / \beta_{\text {coh }}$ measured over the $\mathrm{C} 3$ area of the motor cortex of a right-handed subject for a source-receiver distance of $20 \mathrm{~mm}$. The blue circles are the resting baseline data; the red circles are the data for contralateral stimulation. The error bars are standard deviations from two blocks of ten runs each. Lines are fits of the analytical solution of the three-layer correlation-diffusion Eq. (2) to the data. Best-fit values for diffusion coefficients $D_{1}$ and $D_{3}$ of scalp and cortex, respectively, are $D_{1}=1.0 \times 10^{-9} \mathrm{~cm}^{2} / \mathrm{s}$ and $D_{3}=4.9 \times 10^{-9} \mathrm{~cm}^{2} / \mathrm{s}$ for the resting baseline period, and $D_{1}=1.5 \times 10^{-9} \mathrm{~cm}^{2} / \mathrm{s}$ and $D_{3}=1.3 \times 10^{-8} \mathrm{~cm}^{2} / \mathrm{s}$ for the stimulation period.

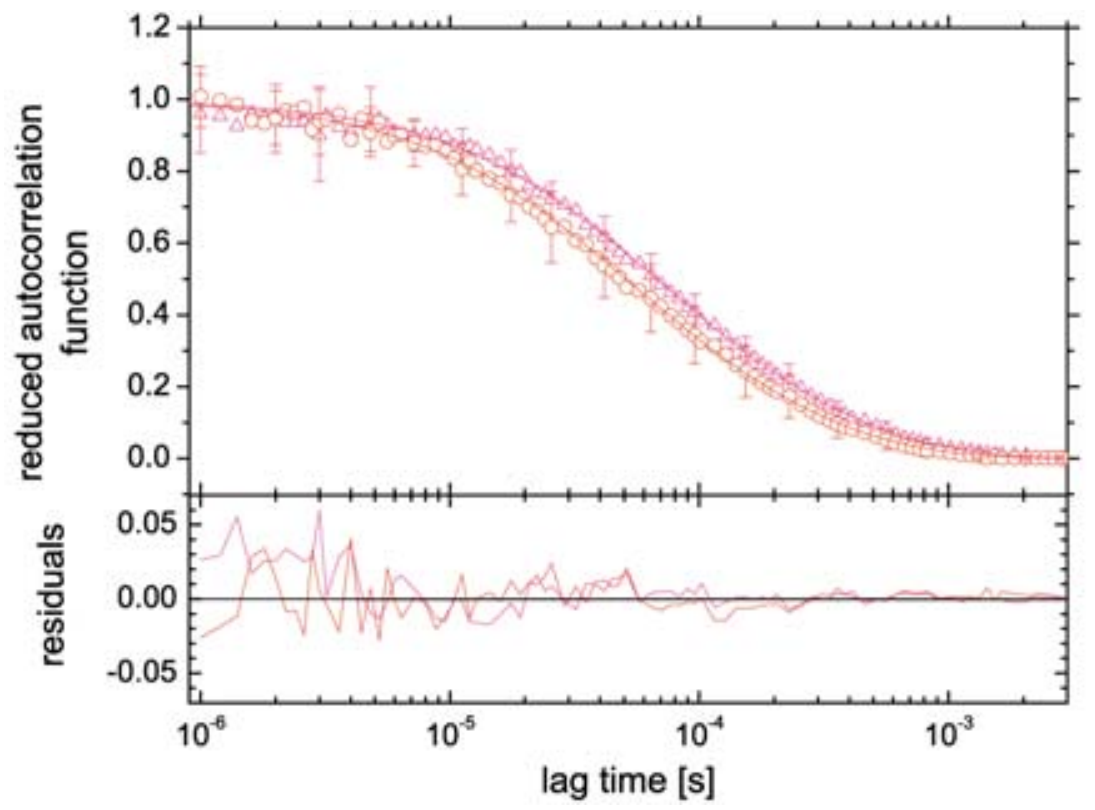

Fig. 3 Reduced intensity autocorrelation function $\left[g^{(2)}(r, \tau)-1\right] / \beta_{\text {coh }}$ measured over the C3 area of the motor cortex of a right-handed subject for a source-receiver distance of $20 \mathrm{~mm}$. The magenta triangles are the data for ipsilateral (left-hand) stimulation; the red circles are the data for contralateral stimulation. The error bars are standard deviations from two blocks of ten runs each. Lines are fits of the analytical solution of the three-layer correlation-diffusion Eq. (2) to the data. Best-fit values for diffusion coefficients $D_{1}$ and $D_{3}$ of scalp and cortex, respectively, are $D_{1}=1.2 \times 10^{-9} \mathrm{~cm}^{2} / \mathrm{s}$ and $D_{3}=9.8 \times 10^{-9} \mathrm{~cm}^{2} / \mathrm{s}$ for ipsilateral stimulation and $D_{1}=1.5 \times 10^{-9} \mathrm{~cm}^{2} / \mathrm{s}$ and $D_{3}=1.3 \times 10^{-8} \mathrm{~cm}^{2} / \mathrm{s}$ for the contralateral stimulation. 


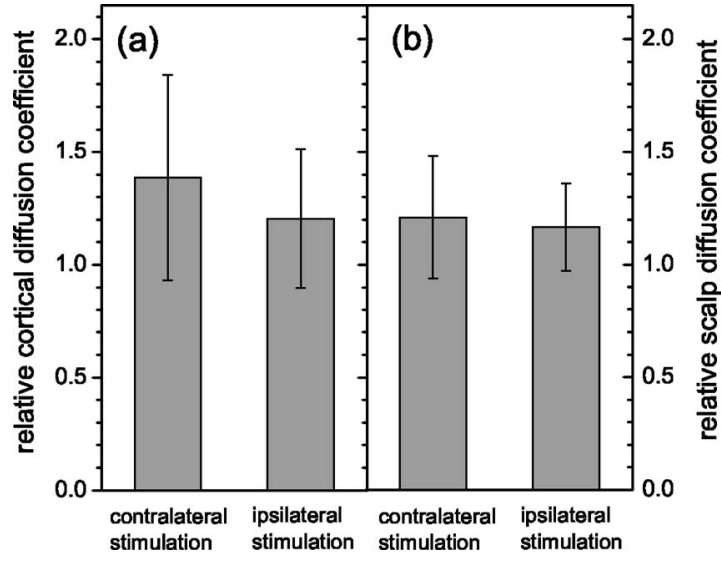

Fig. 4 Diffusion coefficients of (a) the cortex and (b) the scalp, measured over C3 from 11 right-handed subjects during contra- and ipsilateral stimulation, normalized by their respective baseline values. Contralateral stimulation results in a significant increase of the cortical diffusion coefficient $D_{3}$ by $38.6 \%$ [t-test (two-tailed): $t(10)=2.81$, $p=0.018<0.05]$, while the response of $D_{3}$ to ipsilateral stimulation is weaker $(20.4 \%)$ and only weakly significant [t-test (two-tailed): $t(10)=2.20, p=0.052>0.050]$. Motor stimulation results in enhanced peripheral perfusion, reflected by the increased scalp diffusion coefficient $D_{1}$ for both contra- and ipsilateral stimulation: by $20.9 \%$ [t-test (two-tailed): $t(10)=2.55, p=0.029<0.050]$ for contralateral stimulation, and by $16.7 \%$ [t-test (two-tailed): $t(10)=2.86, p=0.017$ $<0.050]$ for ipsilateral stimulation.

periods with the one for the resting period for each subject, reveals that across the subject group the finger opposition task produces a significant increase of the cortical diffusion coefficient $D_{3}$ measured at C3 during contralateral stimulation by $38.6 \%$ [ $t$-test (two-tailed): $t(10)=2.81, p=0.018<0.050]$, as shown in Fig. 4(a). Ipsilateral (left hand) stimulation results in an increase of $D_{3}$ by about $20.4 \%$, which is, however, not as significant as the one observed for contralateral stimulation [ $t$-test (two-tailed): $t(10)=2.20, p=0.052>0.050]$. Direct comparison of the relative cortical diffusion coefficients for contra- and ipsilateral stimulation shows a significant hemispheric asymmetry between the two responses $[t$-test (twotailed): $t(10)=2.69, p=0.023<0.050]$.

Interestingly, the finger opposition task leads also to an increase of the scalp diffusion coefficient $D_{1}$, albeit not as large as the increase of the cortical diffusion coefficient [see Fig. 4(b)]: contralateral stimulation leads to a $20.9 \%$ increase in $D_{1}$ above the baseline value [t-test (two-tailed): $t(10)$ $=2.55, p=0.029<0.050]$, while an increase by about $16.7 \%$ can be seen for ipsilateral stimulation [ $t$-test (two-tailed): $t(10)=2.86, p=0.017<0.050]$. Direct comparison shows, on the other hand, that the difference in $D_{1}$ between contra- and ipsilateral responses is not significant [ $t$-test (two-tailed): $t(10)=0.731, p=0.480>0.050]$.

\section{Discussion}

The observed large spread of the value of $D_{3}$ between subjects might be due to the varying accuracy of localizing C3 using only external landmarks. A combination of DWS and independent measurements of the optical parameters by NIRS (such as in Ref. 32) or time-of-flight methods might help to resolve this issue.
In our experiments with the 11 subjects, in two subjects the response of the cortical diffusion coefficient to ipsilateral stimulation is larger than the one to contralateral stimulation, opposite to what would be expected from EEG data. This anomaly might result from a variant in the location of the contralateral somato-motor hand representation that may not be near the C3-scalp location in these subjects, or from an anomaly in the motor organization, which is known to occur in about $5 \%$ of the subjects. ${ }^{37}$

As the theoretical expressions [Eqs. (16) and (17)] for the three-layer model used for extracting cortical and scalp diffusion coefficients contains ten parameters (layer thickness $\Delta_{1,2}$, absorption path lengths $l_{1,2,3}^{(\mathrm{a})}$, transport mean free path lengths $l_{1,2,3}^{*}$, and diffusion coefficients $D_{1,3}$ ) when the skull is treated as a static medium, it is important to know how robust the determination of $D_{3}, D_{1}$, and $\Delta_{2}$ is with respect to uncertainties in the optical parameters $l_{n}^{(\mathrm{a})}$ and $l_{n}^{*}$ of scalp, skull, and cortex, and in the local thickness of the skin. In particular, an uncontrolled increase in the cortical transport mean free path could be interpreted as a decreased cortical diffusion coefficient. Conversely, a decrease in the cortical absorption coefficient could be interpreted as an increase of the diffusion coefficient. Weaker influences on the determination of the cortical diffusion coefficient are expected from changes in the optical properties of the scalp and skull, since these affect the decay of the autocorrelation function mainly at long times.

We have thus modeled autocorrelation functions with largely varying values of the optical and dynamical parameters using the analytical solution of the correlation-diffusion equation for a three-layer model of the human head, and compared these predictions with measured data. Figure 5(a) shows a plot of the square root of the sum of squared residuals $\chi$ between the measured and calculated intensity autocorrelation functions as a function of $D_{3}$ and $l_{3}^{(\text {a) }}$. Varying the cortical diffusion coefficient from $D_{3}=2.5 \times 10^{-9} \mathrm{~cm}^{2} / \mathrm{s}$ to $D_{3}=1.0$ $\times 10^{-8} \mathrm{~cm}^{2} / \mathrm{s}$ and the cortical absorption length from $l_{3}^{(\mathrm{a})}$ $=1.5 \mathrm{~cm}$ to $l_{3}^{(\mathrm{a})}=6.0 \mathrm{~cm}$, we find that $\chi$ shows a well-defined minimum at the best-fit values $D_{3}=4.9 \times 10^{-9} \mathrm{~cm}^{2} / \mathrm{s}$ and $l_{3}^{(\mathrm{a})}=3.0 \mathrm{~cm}$ for the cortical diffusion coefficient and the cortical absorption length, respectively. However, fits with only slightly higher $\chi$ could be obtained by reducing $D_{3}$ to below $2.5 \times 10^{-9} \mathrm{~cm}^{2} / \mathrm{s}$ and increasing $l_{3}^{(\mathrm{a})}$ to about $5.0 \mathrm{~cm}$. This correlation between $l_{3}^{(\mathrm{a})}$ and $D_{3}$ reflects the fact that higher absorption cuts off long photon paths, which in turn might be interpreted as slowed-down dynamics (i.e., smaller $D_{3}$ ). An overestimation of $l_{3}^{(\mathrm{a})}$ and a concurrent underestimation of $D_{3}$ can occur if the data quality is not sufficient to capture the rounding of $g^{(1)}(\mathbf{r}, \tau)$ at short lag times, which is the hallmark of absorption. ${ }^{48}$ Rather than corresponding to a physically meaningful value, the very large value of the cortical absorption length of $5.0 \mathrm{~cm}$ at the second minimum in $\chi\left(D_{3}, l_{3}^{(\mathrm{a})}\right)$ is more likely to reflect the noise in the data at short lag times.

Bounds for the activation-related changes in $l_{3}^{(\mathrm{a})}$ can be obtained from independent measurements using photon migration or time-of-flight methods. Recent experiments show that the concentration of deoxy-hemoglobin in the motor cortex decreases on activation by up to about $0.2 \mu \mathrm{M}$, while the increased oxygen consumption is reflected by an increase of the oxy-hemoglobin concentration by about $0.6 \mu \mathrm{M}$ over the 
(a)

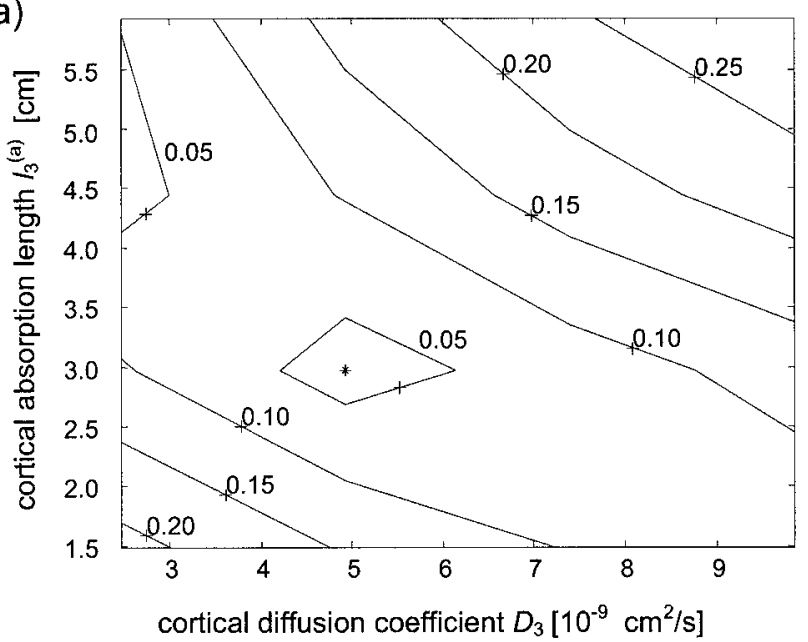

(b)

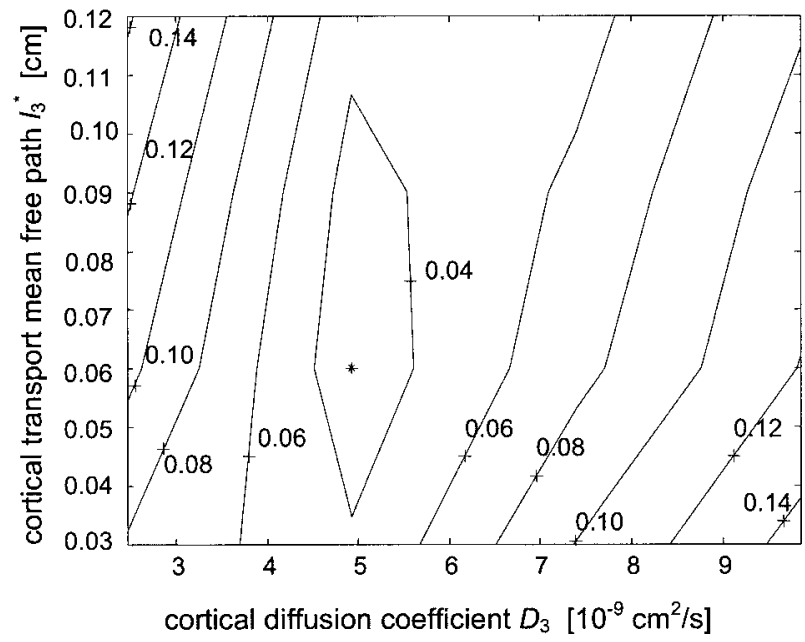

Fig. 5 Plot of the sum of squared residuals $\chi$ between the prediction of the correlation-diffusion Eq. (2) for a three-layer model and experimental baseline data as a function of cortical diffusion coefficient $D_{3}$ and (a) cortical absorption length $I_{3}^{(a)}$, and (b) cortical transport mean free path length $I_{3}^{*}$. Parameters for the calculation are $l_{1}^{(\mathrm{a})}=3.97 \mathrm{~cm}$, $l_{1}^{*}=0.0509 \mathrm{~cm}, \Delta_{1}=0.10 \mathrm{~cm}$ for the scalp; $l_{2}^{(a)}=3.97 \mathrm{~cm}$, $r_{2}^{*}=0.0509 \mathrm{~cm}, \Delta_{2}=0.47 \mathrm{~cm}$ for the skull; and $l_{3}^{(\text {a) }}=2.96 \mathrm{~cm}$, $\Gamma_{3}=0.060 \mathrm{~cm}, \Delta_{3}=\infty$ for the cortex. Diffusion coefficients are $D_{1}=1.0 \times 10^{-9} \mathrm{~cm}^{2} / \mathrm{s}$ for the scalp, $D_{2}=0$ for the skull, and $D_{3}=4.9 \times 10^{-9} \mathrm{~cm}^{2} / \mathrm{s}$ for the brain. The asterisks mark the minimum of $\chi$.

average total hemoglobin concentration of about $74 \mu \mathrm{M} .{ }^{10}$ At the wavelength $\lambda_{0}=802 \mathrm{~nm}$ used in our experiments, which is close to the isosbestic point of oxy-/deoxy-hemoglobin, relative changes in the cortical absorption coefficient $1 / l_{3}^{(\mathrm{a})}$ are thus expected to be given by the changes in the total hemoglobin concentration, which are of the order of $1 \%$ around the baseline level. From our sensitivity plot, however, such changes would lead to changes in the cortical diffusion coefficient of no more than a few percent. On the other hand, we find that the cortical diffusion coefficient measured at $\mathrm{C} 3$ for contralateral stimulation is increased by $38.6 \%$ in the group average, which is clearly not explained by the perfusion-

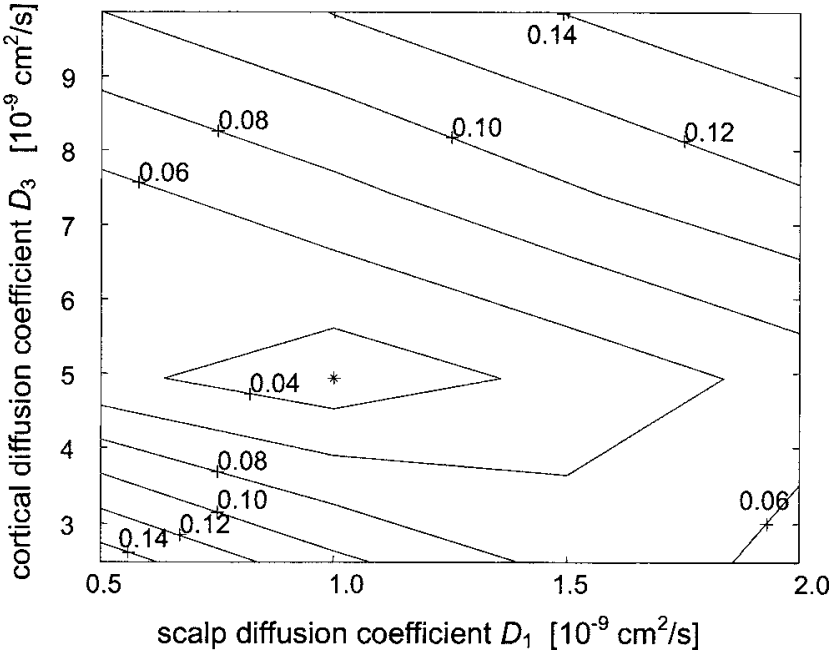

Fig. 6 Plot of $\chi$ between the prediction of the three-layer correlationdiffusion theory and the experimental baseline data as a function of the diffusion coefficients $D_{1}$ and $D_{3}$ of scalp and cortex, respectively. Constant parameters are as in Fig. 5.

related increases in erythrocyte concentration or blood volume.

On the other hand, a plot of $\chi$ as a function of $D_{3}$ and $l_{3}^{*}$ [see Fig. 5(b)] shows that the minimum of $\chi\left(D_{3}, l_{3}^{*}\right)$ around the optimal fit parameter $D_{3}=4.9 \times 10^{-9} \mathrm{~cm}^{2} / \mathrm{s}$ is quite pronounced. Varying the cortical transport mean free path length $l_{3}^{*}$ from $l_{3}^{*}=0.03 \mathrm{~cm}$ to $l_{3}^{*}=0.12 \mathrm{~cm}$ does not significantly affect the value of $\chi$ for the cortical diffusion coefficient fixed at the best-fit value $D_{3}=4.9 \times 10^{-9} \mathrm{~cm}^{2} / \mathrm{s}$. This shows that the determination of the cortical diffusion coefficient from fitting the full three-layer model to measured correlation functions is quite robust against uncertainties in the estimation of the cortical transport mean free path length.

Sensitivity plots of $\chi$ as a function of the cortical diffusion coefficient $D_{3}$ and the optical and dynamical parameters of the scalp show that the behavior of $g^{(1)}(\mathbf{r}, \tau)$ at short times (governed by $D_{3}$ ) is sufficiently well decoupled from its longtime behavior that even sizeable changes in scalp parameters $\Delta_{1}, l_{1}^{(\mathrm{a})}$ and $l_{1}^{*}$ do not influence the determination of $D_{3}$ significantly. Conversely, the determination of the scalp diffusion coefficient $D_{1}$ was found to be largely unaffected by variations in cortical optical parameters.

Finally, the dependence of $\chi$ on $D_{1}$ and $D_{3}$ (Fig. 6) shows a single minimum for a wide range of diffusion coefficients $0.5 \times 10^{-9} \mathrm{~cm}^{2} / \mathrm{s} \leqslant D_{1} \leqslant 2.0 \times 10^{-9} \mathrm{~cm}^{2} / \mathrm{s}$ and 2.5 $\times 10^{-9} \mathrm{~cm}^{2} / \mathrm{s} \leqslant D_{3} \leqslant 1.0 \times 10^{-8} \mathrm{~cm}^{2} / \mathrm{s}$, which is rather flat in the direction of $D_{1}$. This means that the estimate of the cortical diffusion coefficient $D_{3}$ is rather insensitive to uncertainties in value of the scalp diffusion coefficient $D_{1}$.

It should be noted that the same qualitative behavior is shown when $\chi$ is calculated between theory and experimental data recorded during stimulation.

In addition, from the considerations on the robustness of the fitting procedure with respect to uncertainties in optical parameters, the accuracy in determining cortical diffusion coefficients also depends on the noise in the measured data arising from finite photon counting statistics. Noise in correlation 
functions is itself correlated and difficult to estimate. ${ }^{49}$ In general, autocorrelation functions measured with low average photon count rate or fast decay times are particularly prone to errors. To assess the variance of $D_{3}$ arising from noise in the data, we analyzed the data for subject 3 . These data show both a low average count rate of $21 \mathrm{kHz}$, and a short average decay time $\bar{\tau}=38 \mu \mathrm{s} .^{\dagger}$ Analyzing the 20 autocorrelation functions from two blocks individually with fixed coherence factor $\beta_{\text {coh }}=0.075^{\ddagger}$ and fixed skull thickness $\Delta_{2}=5.2 \mathrm{~mm}$ yielded cortical diffusion coefficients $D_{3}=(6.7 \pm 2.2) \times 10^{-9} \mathrm{~cm}^{2} / \mathrm{s}$ during the resting baseline period and $D_{3}=(8.3 \pm 1.7)$ $\times 10^{-9} \mathrm{~cm}^{2} / \mathrm{s}$ for contralateral stimulation. These values are in good agreement with the ones obtained from the analysis of the averaged autocorrelation functions. The analysis of these data shows that with even considerable noise in the measured data, the difference in cortical diffusion coefficients between resting baseline and stimulation periods is highly significant within the datasets from a single subject, at least for contralateral stimulation where the somato-sensory activation can be expected to be maximal.

In modeling our data with a three-layer geometry consisting of scalp, skull, and cortex, we have neglected the cerebrospinal fluid (CSF) layer between skull and cortex, whose absorption and transport mean free path lengths are both much larger than its thickness. Simulations have shown that the presence of the nearly transparent CSF can influence the spatial sensitivity profile or the path length distribution of diffusing photons; ${ }^{46}$ for a thickness exceeding about $1 \mathrm{~mm}$, photon propagation in the presence of the CSF is no longer well described by the diffusion approximation, ${ }^{50}$ which is of particular importance for the accurate inversion of photon migration data. Light guiding by the CSF for large sourcereceiver distances reduces the photon flux through the cortex and, hence, the number of detected photons that can pick up its dynamics. The simulations show, on the other hand, that for the source-receiver distance used in our experiments, light-guiding effects should be small. The marked difference in the short-time behavior of $g^{(1)}(\mathbf{r}, \tau)$ between resting and stimulation periods shows that with the present sourcereceiver distance, enough detected photons have indeed crossed the CSF and passed through the cortex for a measurable activation-related increase of the cortical diffusion coefficient. Surprisingly, the predictions for $g^{(1)}(\mathbf{r}, \tau)$ from the three-layer model in the diffusion approximation show very good agreement with the data, even at the longest times where the effect of the CSF could be expected to be strongest. Indeed, Monte-Carlo simulations of DWS autocorrelation functions for four-layer systems, including a nonscattering CSF layer, show a shape that is very similar to the one for threelayer systems. An analysis of four-layer simulation data with a three-layer model shows that the obtained value of the cortical diffusion coefficient is underestimated only slightly.

Clearly our analysis of the sensitivity of the fitting procedure on uncertainties in the optical and dynamical parameters shows that within realistic bounds for the optical and dynami-

\footnotetext{
${ }^{\dagger}$ We use $\bar{\tau}=\int_{\tau=200 \mathrm{~ns}}^{\tau=3.1 \mathrm{~s}} d \tau\left[g^{(2)}(r, \tau)-1\right] / \beta_{\text {coh. }}$.

${ }^{\ddagger}$ This value of $\beta_{\text {con }}$ results from a combination of the optics of the receiver fiber guiding six transverse modes, and the presence of about two longitudinal laser modes of unequal amplitude, yielding an effective mode number of $2 \times 6=12 \approx 1 / \beta_{\text {coh }}$.
}

cal tissue parameters, the faster decay of the autocorrelation function during a motor task can be assigned to an increase of the cortical diffusion coefficient due to cortical activation. It is, however, not quite clear whether this enhanced dynamic is related to blood flow or to neural dynamics, or due to a combination of both. While the mere increase of blood volume due to activation cannot explain the observed increase in the cortical diffusion coefficient, vasodilation could lead to a large increase in blood flow velocity, even for small increases in blood volume due to the strong decrease in hydrodynamic resistance with the vessel diameter. Interestingly, the measured increase in the cortical diffusion coefficient of $38.6 \%$ for contralateral stimulation is comparable to positron emission tonography (PET) measurements ${ }^{51,52}$ of the functional blood flow increase in motor cortical areas slightly smaller than the ones probed in our DWS experiments. In addition, the functional increase of $D_{3}$ measured in our experiments is in good agreement with the corrected relative blood flow rate increase measured by Durduran et al. using DWS.

On the other hand, the high turbidity of cortical tissue indicates that DWS is sensitive not only to motions of erythrocytes, but also to motions of and within neura. At a hematocrit of $5 \%$, bulk blood has a transport mean free path length $l^{*}=3.8 \mathrm{~mm}$ at $\lambda=800 \mathrm{~nm} .^{53}$ Extrapolation to a physiological hematocrit of $50 \%$ and a cerebral blood volume of $4 \mathrm{~g} / 100 \mathrm{~m} \ell$ yields $l^{*}=9.6 \mathrm{~mm}$, which is more than an order of magnitude larger than the transport mean free path length of typical cortical tissue with $l_{3}^{*} \approx 0.4 \mathrm{~mm},{ }^{44}$ which is dominated by the scattering from neura. Photons diffusing in cortical tissue should thus experience many more scattering events from neura than from erythrocytes. If both neural organelles and erythrocytes move mainly by Brownian motion (which is a reasonable approximation for the short length scales probed by DWS), the effective diffusion coefficient $D_{3}$ measured by DWS is the sum of the individual diffusion coefficients, each weighted with the reduced scattering coefficient of the respective component. ${ }^{48}$ Assuming vesicles with diameter $200 \mathrm{~nm}$, their contribution to $D_{3}$ is expected to dominate over the one from the erythrocytes at a vesicle volume fraction of about $18 \% .^{\S}$ Although this is a rough estimate, this value is consistent with values measured by electron microscopy in chromaffin cells. ${ }^{55}$ While the contribution of subcellular organelle motion to the cortical diffusion coefficient could be investigated by local ischemia on arteriolar occlusion in animal models (similar to the experiments in Ref. 31), such experiments are not permissible in the human brain. However, laser Doppler measurements of biological zero signals (e.g., under cuff compression) in other types of tissue show that subcellular organelle motion not related to perfusion indeed contributes to the speckle fluctuations from perfused tissue. ${ }^{56}$

Similar to the situation at rest, cortical diffusion coefficients measured during activation could be increased not only due to enhanced blood flow rate, but also due to increased mobility of synaptic vesicles associated with primary neural signals. ${ }^{57-59}$ While for the current motor protocol and data

\footnotetext{
${ }^{\S}$ We model the vesicles as spheres with relative dielectric constant $\epsilon_{\mathrm{r}}=1.22$, from which we calculate a scattering cross section $\sigma_{v}=4.7 \times 10^{-2} \mu \mathrm{m}^{2}$ in the Rayleigh approximation. For the erythrocytes we use a scattering cross section $\sigma_{\mathrm{e}}=100 \mu \mathrm{m}^{2},{ }^{54}$ an anisotropy factor $g_{\mathrm{e}}=0.99$, and a number density $\rho_{\mathrm{e}}=5 \times 10^{-3} \mu \mathrm{m}^{-3}$. $^{53}$
} 
collection scheme the perfusion-related dynamics cannot be separated from primary neural signals to the short-time decay of the DWS autocorrelation function, one possible way to look for primary neural signals using DWS is by measuring the temporal intensity autocorrelation function with a shorter integration time. If the DWS signal is due to cerebral hemodynamics, the maximum difference of $D_{3}$ (compared with the baseline measurement) should appear with a delay of several seconds with respect to the onset of stimulation. In contrast, if synaptic vesicle motion also makes a significant contribution to the DWS signal, one should detect changes in the cortical diffusion coefficient earlier, from several tens to hundreds of milliseconds after the beginning of neural activation. To address this question experimentally, visual stimulation protocols with rapid visual serial presentation could be used. ${ }^{60}$ The high temporal resolution of EEG and magnetoencephalography (MEG) revealed that the brain is capable of switching rapidly (within less than $100 \mathrm{~ms}$ ) between stimuli, recognizing the emotional character of each input picture. Obviously the cerebral blood flow cannot follow such rapid changes, so if another fast dynamical process, such as synaptic vesicle motion, also contributes significantly to the DWS signal, distinct signal changes for high-arousing versus low-arousing affective stimuli over parietal (preferably right-hemispheric) areas should be seen with this technique.

\section{Conclusions}

We measure diffusion coefficients in the motor cortex of humans during motor stimulation, by analyzing near-infrared diffusing-wave spectroscopy data with the analytical solution of the correlation-diffusion equation for a three-layer geometry modeling the human head. The three-layer analysis yields good agreement with the experimental data over the entire range of lag times spanning more than three decades, and yields a quantitative estimate for the cortical diffusion coefficient. Analyzing the sensitivity of the model to uncertainties in tissue optical parameters, we find that the cortical diffusion coefficient can be extracted from our data to within a few percent if we allow tissue optical parameters to vary within realistic bounds. Performing motor stimulation experiments on 11 right-handed subjects, the autocorrelation function from the $\mathrm{C} 3$ region is found to decay faster during the stimulation period. This enhancement of the dynamics is shown to arise from changes in the cortical dynamics and not from changes in the optical properties of the tissue; our results thus support the interpretation of earlier DWS results for somato-motor stimulation in terms of functionally enhanced blood flow rate. $^{32}$ The marked hemispheric asymmetry between the measured cortical diffusion coefficients in the $\mathrm{C} 3$ region for leftand right-hand stimulation is consistent with the dominant activation of the contralateral motor and sensory regions in right-handed subjects by unimanual motor tasks. While large hemodynamic changes dominate the cortical diffusion coefficient for the present motor stimulation protocol, detection of speckle fluctuations with shorter integration times might allow us to use DWS for the detection of primary neural signals associated with functionally enhanced motion of subcellular organelles, such as synaptic vesicles.

\section{Acknowledgments}

We thank A. Wille and W. Möbius for experimental work in the early stages of the project, B. Luo and H. Zhang for providing us their Monte-Carlo data, A. Roggan and M. Meinke for providing blood optical data, and J. Hebden, M. Junghöfer, M. Wolf, and A. Yodh for helpful discussions. This work was funded by the Deutsche Forschungsgemeinschaft (DFG), the Optik-Zentrum Konstanz, and the Zentrum für Wissenschaftlicher Nachwuchs (ZWN) at the University of Konstanz.

\section{Appendix 1: Field Autocorrelation Function from an $N$-Layer Turbid Medium}

We consider a turbid medium consisting of $N$ slabs with parallel boundaries located at depths $z=L_{n}(n=1, \ldots, N)$. The front surface of the medium is located at $z=L_{0}=0$. Each slab with thickness $\Delta_{n}=L_{n}-L_{n-1}$ is characterized by its transport mean free path $l_{n}^{*}$ and absorption length $l_{n}^{(\mathrm{a})}(n=1, \ldots, N)$. For each slab, we assume its thickness $\Delta_{n}$ to be much larger than the respective transport mean free path $l_{n}^{*}$. Scatterer dynamics inside each layer are modeled by a diffusion coefficient $D_{n}$. Unlike the treatment for DWS from a multilayer system given before ${ }^{33}$ here we assume that the source of monochromatic light is pointlike, located at $\mathbf{r}^{\prime}=\left\{\boldsymbol{\rho}^{\prime}=0, z^{\prime}\right\}$ inside the first layer.

In the diffusion approximation, the normalized temporal autocorrelation function of the scattered field $g^{(1)}(\mathbf{r}, \tau)$ is obtained from the the correlation-diffusion equation ${ }^{24,28}$

$$
\left[\nabla^{2}-\alpha^{2}(\tau)\right] G(\mathbf{r}, \tau)=-s_{0} \delta\left(\mathbf{r}-\mathbf{r}^{\prime}\right),
$$

for the unnormalized field autocorrelation function $G(\mathbf{r}, \tau)$ $=\left\langle E^{*}(\mathbf{r}, 0) E(\mathbf{r}, \tau)\right\rangle$. In each layer, the loss of correlation due to the motion of the scatterers is given by

$$
\alpha_{n}^{2}(\tau)=\frac{3}{l_{n}^{*} l_{n}^{(\mathrm{a})}}+\frac{6 \tau}{\tau_{n}^{(0)} l_{n}^{* 2}},
$$

where $\tau_{n}^{(0)}=\left(k_{n}^{2} D_{n}\right)^{-1}$ is the single-scattering correlation time and $k_{n}$ is the wavenumber of the light in the $n$ 'th layer.

Equation (2) is solved using the Fourier transform of $G(\mathbf{r}, \tau)$ with respect to the transverse coordinate $\boldsymbol{\rho}$

$$
\hat{G}(\mathbf{q}, z, \tau)=\int \mathrm{d}^{2} \boldsymbol{\rho} G(\mathbf{r}, \tau) \exp (i \mathbf{q} \cdot \boldsymbol{\rho}),
$$

yielding

$$
\left[\frac{\partial^{2}}{\partial z^{2}}-\beta^{2}(\mathbf{q}, \tau)\right] \hat{G}(\mathbf{q}, z, \tau)=-s_{0} \delta\left(z-z^{\prime}\right),
$$

for $\hat{G}(\mathbf{q}, z, \tau)$. Here $\beta_{n}^{2}(\mathbf{q}, \tau)=\alpha_{n}^{2}(\tau)+\mathbf{q}^{2}$.

We divide the first layer into two sublayers: layer 0 $\left(0<z<z^{\prime}\right)$, identified by $n=0$, and layer $1\left(z^{\prime}<z<L_{1}\right)$, identified by $n=1$ in the following. The solution of Eq. (5) inside the $n$ 'th layer $(n=0,1,2, \ldots, N)$ then can be written as 


$$
\hat{G}_{n}(\mathbf{q}, z, \tau)=A_{n} \exp \left(\beta_{n} z\right)+B_{n} \exp \left(-\beta_{n} z\right),
$$

where $A_{n}$ and $B_{n}$ are constant (i.e., $z$ independent) factors obtained from the boundary conditions

$$
\begin{gathered}
\hat{G}_{0}(\mathbf{q}, z, \tau)-z_{0} \frac{\partial}{\partial z} \hat{G}_{0}(\mathbf{q}, z, \tau)=0, \quad z=0, \\
\hat{G}_{0}(\mathbf{q}, z, \tau)=\hat{G}_{1}(\mathbf{q}, z, \tau), \quad z=z^{\prime}, \\
\frac{\partial}{\partial z} \hat{G}_{0}(\mathbf{q}, z, \tau)=\frac{\partial}{\partial z} \hat{G}_{1}(\mathbf{q}, z, \tau)+s_{0}, \quad z=z^{\prime}, \\
\hat{G}_{n}(\mathbf{q}, z, \tau)=\hat{G}_{n+1}(\mathbf{q}, z, \tau), \quad z=L_{n}, \quad n=1, \ldots, N-1, \\
\mathcal{D}_{n} \frac{\partial}{\partial z} \hat{G}_{n}(\mathbf{q}, z, \tau)=\mathcal{D}_{n+1} \frac{\partial}{\partial z} \hat{G}_{n+1}(\mathbf{q}, z, \tau), \\
z=L_{n}, \quad n=1, \ldots, N-1, \\
\hat{G}_{N}(\mathbf{q}, z, \tau)+z_{L} \frac{\partial}{\partial z} \hat{G}_{N}(\mathbf{q}, z, \tau)=0, \quad z=L_{N},
\end{gathered}
$$

where $z_{0} \sim l_{1}^{*}$ and $z_{L} \sim l_{N}^{*}$ are the extrapolation lengths taking into account internal reflections at the external $(z=0$ and $z=L_{N}$, respectively) boundaries of the slab. $\mathcal{D}_{n}=c l_{n}^{*} / 3$ denotes the photon diffusion coefficient in layer $n$, and $c$ is the speed of light.

When Eq. (6) is substituted into Eqs. (7) through (12), we obtain a system of $2(N+1)$ linear algebraic equations for coefficients $A_{n}, B_{n}(n=0,1, \ldots, N)$. The Fourier transform $\hat{G}_{0}(\mathbf{q}, z=0, \tau)$ of the autocorrelation function of diffusely reflected light $G_{0}(\mathbf{r}, \tau)$ measured at the front surface of the slab is then obtained by substituting the resulting $A_{0}, B_{0}$ into Eq. (6). In all cases, $\hat{G}_{0}(\mathbf{q}, z=0, \tau)$ can be expressed as

$$
\hat{G}_{0}(\mathbf{q}, z=0, \tau)=\frac{\text { numerator }}{\text { denominator }} .
$$

We now give explicit expressions for the numerator and the denominator in Eq. (13) for a semi-infinite one-layer medium and our three-layer head model (scalp, skull, and cortex). As a reasonable approximation, in our calculation we consider the third layer (cortex) as a semi-infinite medium.

For the case of a semi-infinite one-layer medium, Eqs. (6) through (12) yield

$$
\begin{gathered}
\text { numerator }=s_{0} z_{0} \exp \left(-\beta z^{\prime}\right), \\
\text { denominator }=1+\beta z_{0} .
\end{gathered}
$$

The analogous solution for the case of a three-layer medium with an infinitely thick third layer $\Delta_{3} \rightarrow \infty$ yields

$$
\begin{aligned}
\text { numerator }= & s_{0} z_{0}\left\{\beta _ { 1 } \mathcal { D } _ { 1 } \operatorname { c o s h } [ \beta _ { 1 } ( \Delta _ { 1 } - z ^ { \prime } ) ] \left[\beta_{2} \mathcal{D}_{2} \cosh \left(\beta_{2} \Delta_{2}\right)\right.\right. \\
& \left.+\beta_{3} \mathcal{D}_{3} \sinh \left(\beta_{2} \Delta_{2}\right)\right]+\beta_{2} \mathcal{D}_{2}\left[\beta_{3} \mathcal{D}_{3} \cosh \left(\beta_{2} \Delta_{2}\right)\right. \\
& \left.\left.+\beta_{2} \mathcal{D}_{2} \sinh \left(\beta_{2} \Delta_{2}\right)\right] \sinh \left[\beta_{1}\left(\Delta_{1}-z^{\prime}\right)\right]\right\}
\end{aligned}
$$

$$
\begin{aligned}
\text { denominator }= & \beta_{2} \mathcal{D}_{2} \cosh \left(\beta_{2} \Delta_{2}\right)\left[\beta _ { 1 } \left(\mathcal{D}_{1}\right.\right. \\
& \left.+\beta_{3} \mathcal{D}_{3} z_{0}\right) \cosh \left(\beta_{1} \Delta_{1}\right)+\left(\beta_{3} \mathcal{D}_{3}\right. \\
& \left.\left.+\beta_{1}^{2} \mathcal{D}_{1} z_{0}\right) \sinh \left(\beta_{1} \Delta_{1}\right)\right]+\left[\beta _ { 1 } \left(\beta_{3} \mathcal{D}_{1} \mathcal{D}_{3}\right.\right. \\
& \left.+\beta_{2}^{2} \mathcal{D}_{2}^{2} z_{0}\right) \cosh \left(\beta_{1} \Delta_{1}\right)+\left(\beta_{2}^{2} \mathcal{D}_{2}^{2}\right. \\
& \left.\left.+\beta_{1}^{2} \beta_{3} \mathcal{D}_{1} \mathcal{D}_{3} z_{0}\right) \sinh \left(\beta_{1} \Delta_{1}\right)\right] \times \sinh \left(\beta_{2} \Delta_{2}\right)
\end{aligned}
$$

The field autocorrelation function $G_{0}(\mathbf{r}, \tau)$ measured at the position $\mathbf{r}=\{\boldsymbol{\rho}, z=0\}$ on the surface of the medium is then obtained from the inverse Fourier transform

$$
G_{0}(\mathbf{r}, \tau)=\frac{1}{(2 \pi)^{2}} \int \mathrm{d}^{2} \mathbf{q} \hat{G}_{0}(\mathbf{q}, z=0, \tau) \exp (-i \mathbf{q} \cdot \boldsymbol{\rho}),
$$

with respect to $\mathbf{q}$. In view of the complicated form of the expressions, this transform is performed numerically. Note that for $\tau=0$, the presented formalism yields analytical expressions for the Fourier transform of the backscattered intensity $G_{0}(\mathbf{r}, \tau=0)=\left\langle\left|E(\mathbf{r}, t)^{2}\right|\right\rangle$ from multilayer systems measurable in a static experiment.

\section{Appendix 2: Inversion of Measured Autocorrelation Functions}

When the coherence factor $\beta_{\mathrm{coh}}$ relating the field autocorrelation function $g^{(1)}(\mathbf{r}, \tau)$ to the measured intensity autocorrelation function $g^{(2)}(\mathbf{r}, \tau)$ cannot be determined directly from the amplitude of $g^{(2)}(\mathbf{r}, \tau)$ at the shortest lag times $\tau$ (such as when the short-time data are very noisy or for strong afterpulsing), this quantity has to be estimated together with the optical and dynamical parameters $\mathbf{p}=\left(l_{n}^{*}, l_{n}^{(\mathrm{a})}, D_{n}, \Delta_{n}\right)$ governing the field autocorrelation function $g^{(1)}(\mathbf{r}, \tau)$ of a dynamic $N$-layer system, using the theory in Appendix 1.

We define the vector $\mathbf{y}=\left(y_{1}, y_{2}, \ldots, y_{N}\right)$ with components $y_{j}=g^{(2)}\left(\mathbf{r}, \tau_{j}\right)-1$ containing the intensity autocorrelation function measured at lag times $\tau_{j}$, and the vector $\mathbf{u}=\left(u_{1}, u_{2}, \ldots, u_{N}\right)$ whose components are given by the calculated field autocorrelation function $u_{j}=\left|g^{(1)}\left(r, \tau_{j}\right)\right|^{2}$. Instead of determining the optical and dynamical parameters $\mathbf{p}$ from a minimization of the sum of squared residuals

$$
\chi^{2}(\mathbf{p})=\left\|\mathbf{y}-\beta_{\mathrm{coh}} \mathbf{u}\right\|^{2},
$$

with floating coherence factor $\beta_{\mathrm{coh}}$, we eliminate the coherence factor from Eq. (19) by pseudoinversion of the Siegert relation $\mathbf{y}=\beta_{\mathrm{coh}} \mathbf{u}$. This allows us to write Eq. (19) as

$$
\chi^{2}(\mathbf{p})=\left\|\mathbf{y}-\left(\mathbf{u}^{+} \cdot \mathbf{y}\right) \mathbf{u}\right\|^{2}=\left\|\mathbf{y}-\frac{\mathbf{u}^{\mathrm{T}} \cdot \mathbf{y}}{\mathbf{u}^{\mathrm{T}} \cdot \mathbf{u}} \mathbf{u}\right\|^{2},
$$

which can be minimized with respect to the parameter vector p using a Levenberg-Marquardt algorithm, now without the need for simultaneous optimization with respect to $\beta_{\text {coh }}$. 


\section{References}

1. V. Tuchin, Tissue Optics. Light Scattering Methods and Instruments for Medical Diagnosis, Vol. TT38 of Tutorial Texts in Optical Engineering, SPIE Press, Bellingham, WA (2000).

2. B. Chance, "Near-infrared images using continuous, phasemodulated, and pulsed light with quantitation of blood and blood oxygenation," Ann. N.Y. Acad. Sci. 838, 29-45 (1998).

3. D. J. Pine, D. A. Weitz, P. M. Chaikin, and E. Herbolzheimer, "Diffusing-wave spectroscopy," Phys. Rev. Lett. 60, 1134-1137 (1988).

4. N. P. Barry, M. J. Cole, M. J. Dayel, K. Dowling, P. M. W. French, S. C. W. Hyde, R. Jones, D. Parsons-Karavassilis, M. Tziraki, M. J. Lever, K. M. Kwolek, D. D. Nolte, M. R. Melloch, M. A. A. Neil, R. Juskaitis, T. Wilson, A. K. L. Dymoke-Bradshaw, and J. D. Hares, "Imaging biological tissue using photorefractive holography and fluorescence lifetime," in Waves and Imaging through Complex Media, P. Sebbah, Ed., pp. 213-234, Kluwer, Dordrecht (2001).

5. J. M. Schmitt, A. Knüttel, and R. F. Bonner, "Measurement of optical properties of biological tissues by low-coherence reflectometry," Appl. Opt. 32, 6032-6042 (1993).

6. D. A. Boas, M. A. O'Leary, B. Chance, and A. G. Yodh, "Scattering and wavelength transduction of diffuse photon density waves," Phys. Rev. E 47, R2999-R3002 (1993).

7. S. R. Arridge and M. Schweiger, "Image reconstruction in optical tomography," Philos. Trans. R. Soc. London 352, 717-726 (1997).

8. M. A. Franceschini, V. Toronov, M. Filiaci, E. Gratton, and S. Fantini, "On-line optical imaging of the human brain with 160-ms temporal resolution," Opt. Express 6, 49-57 (2000).

9. M. A. Franceschini, S. Fantini, J. J. Thompson, J. P. Culver, and D. A. Boas, "Hemodynamic evoked response of the sensorimotor cortex measured non-invasively with near-infrared optical imaging," Psychophysiology 40, 548-560 (2003).

10. M. Wolf, U. Wolf, V. Toronov, A. Michalos, L. A. Paunescu, J. H. Choi, and E. Gratton, "Different time evolution of oxyhemoglobin and deoxyhemoglobin concentration changes in the visual and motor cortices during functional stimulation: A near-infrared spectroscopy study," Neuroimage 16, 704-712 (2002).

11. G. Gratton, P. M. Corballis, E. Cho, M. Fabiani, and D. C. Hood, "Shades of gray matter: Noninvasive optical images of human brain responses during visual stimulation," Psychophysiology 32, 505-509 (1995).

12. M. Wolf, U. Wolf, H. J. Choi, R. Gupta, L. P. Safanova, L. A. Paunescu, A. Michalos, and E. Gratton, "Functional frequency-domain near-infrared spectroscopy detects fast neuronal signal in the motor cortex," Neuroimage 17, 1868-1875 (2002).

13. M. A. Franceschini and D. A. Boas, "Noninvasive measurement of neuronal activity with near-infrared optical imaging," Neuroimage 21, 372-386 (2004).

14. A. Grinvald, E. Lieke, R. D. Frostig, C. D. Gilbert, and T. N. Wiesel, "Functional architecture of cortex revealed by optical imaging of intrinsic signals," Nature 324, 361-364 (1986).

15. A. Grinvald, L. Anglister, J. A. Freeman, R. Hildesheim, and A. Manker, "Real-time optical imaging of naturally evoked electrical activity in intact frog brain," Nature 308, 848-850 (1984).

16. L. B. Cohen, R. D. Keynes, and B. Hille, "Light scattering and birefringence changes during nerve activity," Nature 218, 438-441 (1968).

17. R. A. Stepnoski, A. LaPorta, F. Raccuia-Behling, G. E. Blonder, R. E. Slusher, and D. Kleinfeld, "Noninvasive detection of changes in membrane potential in cultured neurons by light scattering," Proc. Natl. Acad. Sci. U.S.A. 88, 9382-9386 (1991).

18. A. K. Dunn, H. Bolay, M. A. Moskowitz, and D. A. Boas, "Dynamic imaging of cerebral blood flow using laser speckle," J. Cereb. Blood Flow Metab. 21, 195-201 (2001).

19. H. Bolay, U. Reuter, A. K. Dunn, Z. Huang, D. Boas, and M. A. Moskowitz, "Intrinsic brain activity triggers trigeminal meningeal afferents in a migraine model," Nat. Med. 8, 136-142 (2002).

20. A. K. Dunn, A. Devor, H. Bolay, M. L. Andermann, M. A. Moskowitz, A. M. Dale, and D. A. Boas, "Simultaneous imaging of total cerebral hemoglobin concentration, oxygenation, and blood flow during functional activation," Opt. Lett. 28, 28-30 (2003).

21. T. Durduran, M. G. Burnett, G. Yu, C. Zhou, D. Furuya, A. G. Yodh, J. A. Detre, and J. H. Greenberg, "Spatiotemporal quantification of cerebral blood flow during functional activation in rat somatosensory cortex using laser-speckle flowmetry," J. Cereb. Blood Flow Metab.
24, 518-524 (2004)

22. G. Maret and P. E. Wolf, "Multiple light scattering from disordered media: The effect of Brownian motion of scatterers," Z. Phys. B: Condens. Matter 65, 409-413 (1987).

23. X. L. Wu, D. J. Pine, P. M. Chaikin, J. P. Huang, and D. A. Weitz, "Diffusing-wave spectroscopy in a shear flow," J. Opt. Soc. Am. B 7, 15-20 (1990).

24. D. Bicout and R. Maynard, "Diffusing wave spectroscopy in inhomogeneous flows," Physica A 199, 387-411 (1993).

25. D. Bicout and G. Maret, "Multiple light scattering in Taylor-Couette flow," Physica A 210, 87-112 (1994).

26. M. Heckmeier and G. Maret, "Visualization of flow in multiplescattering liquids," Europhys. Lett. 34, 257-262 (1996).

27. M. Heckmeier, S. E. Skipetrov, G. Maret, and R. Maynard, "Imaging of dynamic heterogeneities in multiple scattering media," J. Opt. Soc. Am. A 14, 185-191 (1997).

28. D. A. Boas, L. E. Campbell, and A. G. Yodh, "Scattering and imaging with diffusing temporal field correlations," Phys. Rev. Lett. 75, 1855-1858 (1995).

29. R. Lohwasser and G. Soelkner, "Experimental and theoretical laserDoppler frequency spectra of a tissue-like model of a human head with capillaries," Appl. Opt. 38, 2128-2137 (1999).

30. C. Cheung, J. P. Culver, K. Takahashi, J. H. Greenberg, and A. G. Yodh, "In vivo cerebrovascular measurement combining diffuse nearinfrared absorption and correlation spectroscopies," Phys. Med. Biol. 46, 2053-2065 (2001).

31. J. P. Culver, T. Durduran, D. Furuya, C. Cheung, J. H. Greenberg, and A. G. Yodh, "Diffuse optical tomography of cerebral blood flow, oxygenation, and metabolism in rat during focal ischemia," J. Cereb. Blood Flow Metab. 23, 911-923 (2003).

32. T. Durduran, G. Yu, M. G. Burnett, J. A. Detre, J. H. Greenberg, J. Wang, C. Zhou, and A. G. Yodh, "Diffuse optical measurement of blood flow, blood oxygenation, and metabolism in a brain during sensorimotor cortex activation," Opt. Lett. 29, 1766-1768 (2004).

33. S. E. Skipetrov and R. Maynard, "Dynamic multiple scattering of light in multilayer turbid media," Phys. Lett. A 217, 181-185 (1996).

34. E. R. Kandel, J. H. Schwartz, and T. M. Jessell, Principles of Neural Science, 4th ed., McGraw-Hill, New York (2000).

35. G. W. Goerres, M. Samuel, I. H. Jenkins, and D. J. Brooks, "Cerebral control of unimanual and bimanual movements: An $\mathrm{H}^{15} \mathrm{O}_{2}$ PET study," NeuroReport 9, 3631-3638 (1998).

36. L. C. Hartlage and R. Gage, "Unimanual performance as a measure of laterality," Neuropsychol Rev. 7, 143-156 (1997).

37. B. Kolb and I. Q. Whishaw, Fundamentals of Human Neuropsychology, 5th ed., Freeman, San Francisco (2002).

38. R. Oldfield, "The assessment and analysis of handedness: The Edinburgh Inventory," Neuropsychologia 9, 97-113 (1971).

39. T. Gisler, H. Rüger, S. U. Egelhaaf, J. Tschumi, P. Schurtenberger, and J. Rička, "Mode-selective dynamic light scattering: theory versus experimental realization," Appl. Opt. 34, 3546-3553 (1995).

40. Deutsche Industrie-Norm EN 60825-1: Sicherheit von LaserEinrichtungen, Deutsches Institut f. Normung e. V., Beuth, Berlin (2003).

41. H. H. Jasper, "The ten-twenty electrode system of the International Federation," Electroencephalogr. Clin. Neurophysiol. 10, 370-375 (1958).

42. I. Flammer and J. Rička, "Dynamic light scattering with single-mode receivers: partial heterodyning regime," Appl. Opt. 36, 7508-7517 (1997).

43. B. J. Berne and R. Pecora, Dynamic Light Scattering, Krieger, Malabar (1990).

44. P. van der Zee, Measurement and Modelling of the Optical Properties of Human Tissue in the Near-Infrared, PhD Thesis, University College, London (1992).

45. S. J. Matcher, M. Cope, and D. T. Delpy, "In vivo measurements of the wavelength dependence of tissue-scattering coefficients between 760 and $900 \mathrm{~nm}$ measured with time-resolved spectroscopy," Appl. Opt. 36, 386-396 (1997).

46. E. Okada, M. Firbank, M. Schweiger, S. R. Arridge, M. Cope, and D. T. Delpy, "Theoretical and experimental investigation of nearinfrared light propagation in a model of the adult head," Appl. Opt. 36, 21-31 (1997).

47. D. A. Boas, J. P. Culver, J. J. Stott, and A. K. Dunn, "Three dimensional Monte Carlo code for photon migration through complex heterogeneous media including the adult human head," Opt. Express 10, 
159-170 (2002).

48. D. J. Pine, D. A. Weitz, J. X. Zhu, and E. Herbolzheimer, "Diffusingwave spectroscopy: dynamic light scattering in the multiple scattering limit," J. Phys. (France) 51, 2101-2127 (1990).

49. K. Schätzel, "Noise in photon correlation and photon structure functions," Opt. Acta 30, 155-166 (1983).

50. H. Dehghani, D. T. Delpy, and S. R. Arridge, "Photon migration in non-scattering tissue and the effects on image reconstruction," Phys. Med. Biol. 44, 2897-2906 (1999).

51. P. E. Roland, B. Larsen, N. A. Lassen, and E. Skinhøj, "Supplementary motor area and other cortical areas in organization of voluntary movements in man," J. Neurophysiol. 43, 118-136 (1980).

52. R. J. Seitz and P. E. Roland, "Learning of sequential finger movements in man: A combined kinematic and positron emission tomography (PET) study," Eur. J. Neurosci. 4, 154-165 (1992).

53. A. Roggan, M. Friebel, K. Dörschel, A. Hahn, and G. Müller, "Optical properties of circulating human blood in the wavelength range 400-2500 nm," J. Biomed. Opt. 4(1), 36-46 (1999).

54. M. Hammer, D. Schweitzer, B. Michel, E. Thamm, and A. Kolb, "Single scattering by red blood cells," Appl. Opt. 37, 7410-7418
(1998).

55. H. Plattner, A. R. Artalejo, and E. Neher, "Ultrastructural organization of bovine chromaffin cell cortex - analysis by cryofixation and morphometry of aspects pertinent to exocytosis," J. Cell Biol. 139, 1709-1717 (1997)

56. D. P. Kernick, J. E. Tooke, and A. C. Shore, "The biological zero signal in laser doppler fluximetry-origins and practical implications," Eur. J. Physiol. 437, 624-631 (1999).

57. D. B. Sattelle, D. J. Green, and K. H. Langley, "Laser light scattering from a neurosecretory tissue," Biol. Bull. 149, 445 (1975).

58. D. B. Sattelle, "Quasielastic laser light scattering and laser doppler electrophoresis as probes of synaptic and secretory terminal function," J. Exp. Biol. 139, 233-252 (1988).

59. D. Englert and C. Edwards, "Effect of increased potassium concentrations on particle motion within a neurosecretory structure," Proc. Natl. Acad. Sci. U.S.A. 74, 5759-5763 (1977).

60. M. Junghöfer, M. Bradley, T. Elbert, and P. Lang, "Fleeting images: A new look at early emotion discrimination," Psychophysiology 38, 175-178 (2001). 